\title{
The Influence of Traffic Networks on the Supply-Demand Balance of Tourism: A Case Study of Jiangsu Province, China
}

\author{
Tao Chen \\ Institute of Geographic Sciences and Natural Resources Research, CAS, Beijing 100101, China \\ Correspondence should be addressed to Tao Chen; chent.10b@igsnrr.ac.cn
}

Received 25 February 2014; Accepted 28 April 2014; Published 26 May 2014

Academic Editor: Caihong Li

Copyright ( 2014 Tao Chen. This is an open access article distributed under the Creative Commons Attribution License, which permits unrestricted use, distribution, and reproduction in any medium, provided the original work is properly cited.

\begin{abstract}
The purpose of this research is to address the impact of traffic networks on the supply-demand balance of tourism and to determine if it is necessary to incorporate the traffic flow data for nodes to determine the significant influences and impacts of traffic networks on tourism. For this purpose, a road network was established for Jiangsu province, and the topological parameters of this network and the tourism degree of coordination among each prefectural city were calculated as well. The results demonstrate that the inclusion of the spatial structure of the traffic network was not necessary for determining the supply-demand balance for tourism; thus, the collection of traffic flow data is required to perform further research. As a side result, it has been determined that the circuit routes are relatively absent from the Jiangsu traffic network, which might hinder tourism, and tourism resources are undersupplied to most prefectural cities in Jiangsu.
\end{abstract}

\section{Introduction}

Tourism is a low pollution and low carbon emission industry with a high profitability margin. The provision of tourism services has become a highly popular choice for those who seek economic improvement and transition [1], and the study of the advantages and impacts of tourism is a popular research subject [2-4]. The healthy development of tourism requires practical measures to maintain a supply-demand balance $[5,6]$, which is closely related to the development of local infrastructure.

To measure this supply-demand balance, the degree of coordination is assessed. This concept is derived from synergetics, which was first introduced by Haken [7] in 1971. Synergetics suggests that whether a system is undergoing a phase transition is determined by its external control parameters and that the transition itself is driven by the tendency to self-organize. The degree to which a macrosystem "selforganizes" describes the reduction in the "degree of freedom" or entropy and the increase in "order" or pattern of the macrosystem. Haken expanded this concept a decade later [8, 9]. Consequently, the degree of coordination has been used to describe the "degree" of a system's self-organizing tendency and thus provide a numerical measurement to characterize the synergetics of a system [10].

The degree of coordination is usually used to measure changes in the patterns of two or more subsystems. Hence, it is crucial to choose relevant and essential subsystems. As Gunn and Var [11] first introduced the concept of a "Tourism System," their tourism system model was composed of two subsystems, supply and demand. In 2002, her new model further emphasized the importance of these two subsystems and the interactions between them. $\mathrm{Wu}$ [12] has also provided a more detailed definition of the relationship between the supply and demand in the tourism model.

In Wu's tourism model, a third subsystem was separated from supply and demand: the transportation system, mainly in terms of the system's modes of transportation and traffic. However, how the transportation subsystem affects the entire tourism system has not been studied extensively. Bieger and Wittmer [13] examined the relationship between airline traffic and tourism, and Culpan [14] divided the tourism system into its demand, marketing, transportation, and accommodations components to assess the impacts of tourism on a developing economy. Prideaux [15] also investigated the influence of the transportation system on the relationship between a 
tourism-generating region and the destination region or the demand and supply systems, respectively.

Such research rarely considers the spatial attributes of a traffic system; yet, the layout and connection of a traffic network should be important determinants of the development of tourism and the degree of coordination within the network. Many tourism studies have provided traffic planning advice based solely on the requirements of the tourism industry, but to accurately assess the relationship between the supply-demand balance of tourism and the existing structure of the traffic network, the characterization of the entering and exiting traffic at each node in the network is required. A network in which each node is given a pair of inlet and outlet traffic flow values can be used to assess the relationships that impact the supply-demand balance of tourism and to evaluate and direct the expansion of the traffic network. In major cities, such data can be acquired from the surveillance systems present at each city entrance. However, in small towns and villages, such data will be hard to acquire.

Before investing massive efforts and funds into the acquisition of traffic flow data, a question must first be addressed: is a traffic network itself already significantly related to the supply-demand balance of tourism? If it is, then traffic flow data are not actually required. A system of traffic network attributes must, thus, be introduced into the analysis in this situation. Hagget et al. $[16,17]$ have provided a method for network analysis that has been widely used and is based on the spatial analysis of resources [18] or on city landscape analysis [19]. Bagler [20] used a more complex method to topologically analyze the airport network of India as well as a worldwide airport network. Pearce [21] studied the spatial patterns of package tourism in Europe, and Weng and Yang [22] used an asymmetric, monopolistic competition mode to examine the spatial structure of a tourism system as well as to provide advice. Lu et al. [23] also implemented a nonplanar topological method for examining a traffic network. Beuthe et al. [24] used a matrix model to estimate the demands of different transportation modes. Finally, Entwisle et al. [25] even used a road, spatial network analysis to characterize the accessibility of family planning in Thailand. However, for this case study, the use of Haggett's method is sufficient.

As for the degree of coordination, it has been mostly used to measure the developing equilibrium among multiple, related systems within disciplines ranging from engineering to economic analyses to environmental studies. Zhang et al. [26] created an urban population, economy, and environment model for Shenyang that includes their degree of coordination, and Li et al. [27] also used a similar coupling model to investigate the coordination between urbanization and the environment. Wang et al. [28] used a coordination model when examining the ecoeconomic system of the Yangtze River Delta. With minor adjustments and proper parameter selection, this method can be used to estimate the development of tourism in a study area.

\section{Study Area}

Jiangsu province is located at the center of the Chinese east coast. To its east is the Yellow Sea, and Shandong province lies to the north. The province of Anhui is located to the west. Zhejiang province and Shanghai city are located to the south-southeast. Jiangsu province stretches from $116^{\circ} 18^{\prime} \mathrm{E}$ to $121^{\circ} 57^{\prime} \mathrm{E}$ and from $30^{\circ} 45^{\prime} \mathrm{N}$ to $30^{\circ} 20^{\prime} \mathrm{N}$ with a total area of $102,600 \mathrm{~km}^{2}$. The province contains 13 prefecture-level cities, with a population of approximately 78 million; it is considered one of the most crowded provinces in China. The climates of Jiangsu include warm temperate monsoon and subtropical monsoon, and the Huai River functions as the boundary. The rainfall is concentrated in the summer. The average annual temperature ranges between 13.6 and $16.1^{\circ} \mathrm{C}$. The average annual rainfall is approximately $704-1250 \mathrm{~mm}$, and the mean annual hours of sunshine are 1816-2503 h. Jiangsu is one of the most economically developed provinces in China, as well as one of the provinces with the highest density of traffic infrastructure. Jiangsu has over 3000 years of history starting with the foundation of the vassal state Wu during the Zhou dynasty. Its provincial capital Nanjing served as the national capital for nine dynasties and left a rich and storied legacy. Seven imperial tombs from the Ming dynasty and nine classical Suzhou gardens are recognized as UNESCO World Heritage Sites. In 2010, the revenue from tourism was 468 billion Yuan, which ranked first among all Chinese provinces.

\section{Materials and Methods}

This research aims to establish a traffic network, define the topological index of the network, calculate the supplydemand balance of tourism, and determine if further research concerning the traffic flow of the network is needed.

3.1. Material. In this research, two groups of data are used. One is the spatial vector data for the cities/towns/villages and roads within study area in the year 2005. In that year, the tourism industry was still developing and tended to be more split among different regions. The other group of data is the corresponding statistical data characterizing the degree of coordination for the development of tourism in an area. The $1: 1,000,000$ vector data for the towns and roads of Jiangsu province was acquired from the Chinese National Science and Technology Infrastructure Center, which contains 1578 cities/towns/villages and 2084 roads of various levels. The statistical data were obtained primarily from the Jiangsu Statistical Yearbook, though some data were collected from the China Statistical Yearbook and the Yearbook of China Tourism Statistics. The detailed statistics are listed in Table 1. The A-class tourism attractions were approved by the Chinese National Tourism Administration and ranked from class A to class AAAA by the year 2005. When calculating supply indicator scores, the number of "A"s was used to weigh the number of tourism attractions; a class $\mathrm{A}$ attraction received a score of 1 , while a class AAAA attraction received a 4 .

\subsection{Methods}

\subsubsection{Traffic Topological Network}

(1) Network Construction. Before its use in calculations, vector data must be processed, following the procedure below. 
TABLE 1: Supply and demand system indicators.

\begin{tabular}{ll}
\hline Supply indicator & Demand indicator \\
\hline $\begin{array}{l}\text { Number of A-class } \\
\text { tourism attractions }\end{array}$ & Prefectural city population \\
$\begin{array}{l}\text { Number of local travel } \\
\text { agencies }\end{array}$ & $\begin{array}{l}\text { Income per capita in rural/urban regions } \\
\text { of prefectural cities } \\
\text { Inbound/domestic tourism statistics in } \\
\text { province }\end{array}$ \\
\hline
\end{tabular}

(a) Each city/town/village is considered a node in the network that is attached to the nearest road, unless there is no county level or higher-level road nearby. Due to roads being always built close to human settlements, most nodes can be found significantly close to one road than any other.

(b) If there is a road that connects two nearby towns and does not pass a third town, a line is drawn between the two towns to represent the fact that these two towns are connected by a road route. The end of a road at a province boundary is also used to connect nodes to other provinces but not to connect these provinces to the node itself.

(c) Multiple routes that partially share the same road are still considered as discrete connections because they serve different traveling purposes.

(d) Unlike the network used by Wei and Jia [18] and Qi and Xiong [19], connection lines are allowed to intersect because crossroads are not even close in terms of their importance as towns; thus, intersections are not considered nodes, and each line represents only one route.

(e) If the distances of a node to both roads of a crossroad have the same order of magnitude, it is considered to occur at the crossroad, and connection lines will radiate outwards unlike the triangular or rectangular connections of a normal crossroad.

(f) There are 4 levels of highways as well as railways in the area. Railways are considered as connections only when there is no other road connecting between two adjacent nodes. Expressway has a higher priority than railway but is still not used as connection if possible. National highways, provincial highways, and county roads are treated as the same when counting connection route.

(2) Measurement of the Network Structure. The network structure can be measured using 4 indices, namely, the corridor density, the network connectivity, the point-line ratio, and the degree of network closure.

The corridor density can be defined as the density of connection routes, as in the following formula:

$$
\mathrm{Dc}=\frac{\mathrm{Lr}}{\mathrm{Ar}} \text {. }
$$

$\mathrm{Dc}$ is corridor density, $\mathrm{Lr}$ is the overall length of road in the region, and Ar is the area of region.
The degree of network closure represents the frequency of circuits in the network, the ratio of actual circuits, and maximum possible circuits. This index is named $\alpha$. Consider

$$
\alpha=\frac{(L-N+1)}{(2 N-5)},
$$

where $L$ is the actual number of connections and $N$ is the actual number of nodes in the network, $\alpha \in[0,1]$. A value of $\alpha=0$ signifies that there is no circuit within the network at all, and $\alpha=1$ represents the maximum number of circuits [16].

The $\beta$ index, also known as the topological structure index, describes the point-line ratio; it indicates the degree of difficulty for connecting one node to another. Consider

$$
\beta=\frac{L}{N} .
$$

$L$ and $N$ are defined above, $\beta \in[0,3]$. A higher $\beta$ value indicates a more complicated network [17].

The network connectivity, or $\gamma$ index, is the ratio of the number of existing connections in the network to the maximum possible number of connections, as in the following formula:

$$
\gamma=\frac{L}{3(N-2)} .
$$

$L$ and $N$ are defined above, $\gamma \in[0,1]$. A value of $\gamma=0$ signifies that none of the nodes are connected, and a value of $\gamma=1$ indicates that every node in the network is connected to other nodes [19].

3.2.2. The Degree of Coordination. In synergetics, a parameter that determines whether the system will increase the degree of order of the system is called an order parameter. The contribution of an order parameter to the degree of order is called its efficiency coefficient. A function that describes an efficiency coefficient is called an efficiency function. The efficiency function of an order parameter ranges between 0 and 1 . According to

$$
\begin{array}{r}
E=\frac{X_{i}-\beta_{i}}{\alpha_{i}-\beta_{i}} \quad \text { when } E \text { is positive, } \\
\text { or } E=\frac{\alpha_{i}-X_{i}}{\alpha_{i}-\beta_{i}} \quad \text { when } E \text { is negative, }
\end{array}
$$

where $E$ is the efficiency coefficient. The term $X_{i}$ is the current value of the order parameter, while $\alpha_{i}$ and $\beta_{i}$ are the maximum and minimum values of that order parameter, respectively. Clearly, $E \in[0,1]$. In this research, all the indicators are positive to the system. Hence, only formula (5) is required.

It is easy to determine that this process is actually a standardization that cancels out the units of the quantitative data but still preserves the contribution of the data to the calculation of the "efficiency" of a system.

To describe the overall degree of order for a subsystem, the geometric mean of all the order parameters for one 
subsystem can be used. In this calculation, the maximum and minimum values can be increased and decreased by $1 \%$, respectively, to change the value extent from $E \in[0,1]$ to DO $\in(0,1)$ and comply with the prerequisite that geometric mean is greater than 0 .

Thus, the overall degree of order, or DO, for a subsystem is described by the following formula:

$$
\mathrm{DO}=\sqrt[n]{\prod_{i=1}^{n} E_{i}}, \quad i=1,2, \ldots, n .
$$

Defining the subsystems of supply and demand as $\mathrm{DO}_{S}$ and $\mathrm{DO}_{R}$, we expect the minimum deviation from the two to be

$$
\begin{aligned}
\mathrm{Cv} & =\frac{S}{(1 / 2)\left[\mathrm{DO}_{S}+\mathrm{DO}_{R}\right]} \\
& =2 \sqrt{\left\{1-\frac{\mathrm{DO}_{S} \cdot \mathrm{DO}_{R}}{\left[\left(\mathrm{DO}_{S}+\mathrm{DO}_{U}\right) / 2\right]}\right\}},
\end{aligned}
$$

where $\mathrm{Cv}$ represents the coefficient of variation and $S$ is the standard deviation. Thus, $\sqrt{\mathrm{DO}_{S} \cdot \mathrm{DO}_{R}} \leq\left(\mathrm{DO}_{S}+\mathrm{DO}_{U}\right) / 2$ and, if the $\mathrm{Cv}$ is small, $\sqrt{\mathrm{DO}_{S} \cdot \mathrm{DO}_{R}}$ approaches $\left(\mathrm{DO}_{S}+\right.$ $\left.\mathrm{DO}_{U}\right) / 2$. That is to say,

$$
C=\frac{\sqrt{\mathrm{DO}_{S} \cdot \mathrm{DO}_{R}}}{\left[\left(\mathrm{DO}_{S}+\mathrm{DO}_{U}\right) / 2\right]},
$$

where $C \in[0,1]$ and $C$ represents the degree of coupling. The higher the value of $C$, the better the coupling between two subsystems [27].

However, the degree of coupling does not differentiate the innate difference between two subsystems in terms of whether the systems are well developed or poorly developed. The coupling degree can be high even if the two have a similar degree of development, which can be misleading in terms of the supply-demand balance. Hence, the degree of coupling coordination is introduced to solve this problem:

$$
\begin{gathered}
D=\sqrt{C \cdot T}, \\
T=a \cdot \mathrm{DO}_{S}+b \cdot \mathrm{DO}_{R} .
\end{gathered}
$$

$D$ is the degree of coupling coordination. $T$ is a comprehensive, evaluation coefficient for the supply-demand balance of tourism, and $a$ and $b$ are weights that, in this case, consider the supply and demand to be equally important to tourism and are thus both designated as 0.5 .

\section{Results and Discussion}

4.1. Traffic Network. After the connection network is constructed, the nodes are allocated to the 13 prefectural cities as appropriate for where they belong, along with the connection lines. The overall connection map can be found in Figure 1. The different sizes of the nodes represent the identities as a prefectural capital, city, or town/village. A straight line is a connection route, and the dashed lines are the boundaries that separate towns from different prefectural cities (although these are not necessarily the exact city boundaries).

When calculating numbers of lines and nodes, any line that crosses a border is included in the number of connections present in both prefectural cities. This is because the same route functions as a connection in both provinces, and there is no need to distinguish partial or full connection considering they function identically. The results from these calculations can be found in Table 2 .

The 3 indices appear to be following a similar pattern. The $\alpha$ index indicates that the circuits levels in the province of Jiangsu are basically below average; even the provincial capital, Nanjing, barely attains a value of 0.5 , which means that there is only one route to reach a certain destination for more than half of the possible connection scenarios, and the traveler will have to take the same road when returning to Nanjing. The $\beta$ and $\gamma$ indices are much higher, indicating that it is relatively easy to find a direct path to nearby node. There are two cities, Changzhou and Zhenjiang, that have an average road density but also the highest network indices, presumably due to their low node quantities. This result indicates that the distance between two nodes and the road density are as important as topological indices for the proper description of a traffic network. However, due to the fact that these results are restricted within the province which has "highest road density," it is safe to presume that these indices in other provinces should be much lower.

4.2. Tourism Supply-Demand Coordination. Using the method described previously, the supply and demand indices ( $C$ and $D$, resp.) were calculated for each city, producing a classification system that is divided into 5 levels. The levels are basically divided symmetrically according to actual coordination value of municipality. This classification system is presented in Table 3.

The results describing the degree of coordination and the coordination classification of municipalities are presented in Table 4

There is 1 very low city, 4 low cities, 5 medium cities, 1 high city, and 2 very high cities. Six regions are undersupplied, 4 regions are balanced, and 3 regions are oversupplied, indicating that during a period of rapid development, the affluence of residents increase their affordability, while tourism infrastructures, promotions, and services usually fall behind this increase in wealth, with the exception of provincial "tourism brands" for which tourists from all over the country flock together, or cities with exceptional tourism resources that are not yet exploited to a significant extent.

4.3. Relationship between the Traffic Network and SupplyDemand Coordination. Regression analysis was applied using the network indices and the coordination degrees to assess whether the network structures are already correlated with the degree of coordination. This analysis, performed using SPSS, revealed that a quadratic equation with the network indices as independent variables and the degree of coordination as the dependent variable provided the best fit. It is logical that road conditions can affect tourism, but the 


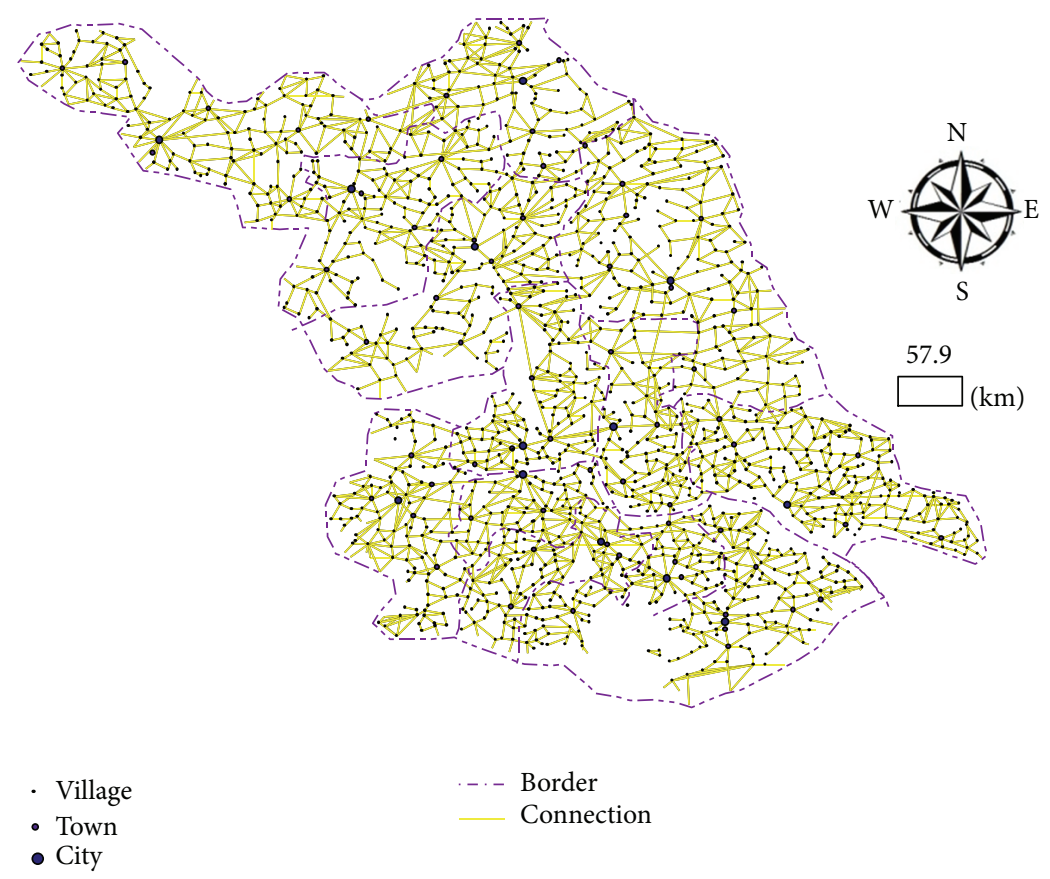

FIgURE 1: Nodes and connections within Jiangsu province.

TABLE 2: Traffic network indices.

\begin{tabular}{|c|c|c|c|c|c|c|}
\hline Municipality & Connection & Node & Corridor density $\left(\mathrm{km} / \mathrm{km}^{2}\right)$ & $\alpha$ & $\beta$ & $\gamma$ \\
\hline Nanjing & 203 & 103 & 0.98 & 0.50 & 1.97 & 0.67 \\
\hline Wuxi & 163 & 93 & 0.88 & 0.39 & 1.75 & 0.60 \\
\hline Xuzhou & 281 & 145 & 0.90 & 0.48 & 1.94 & 0.66 \\
\hline Changzhou & 183 & 85 & 0.81 & 0.60 & 2.15 & 0.73 \\
\hline Suzhou & 223 & 131 & 0.73 & 0.36 & 1.70 & 0.58 \\
\hline Nantong & 348 & 202 & 0.88 & 0.37 & 1.72 & 0.58 \\
\hline Lianyungang & 165 & 90 & 0.60 & 0.43 & 1.83 & 0.63 \\
\hline Huai'an & 212 & 133 & 0.66 & 0.31 & 1.59 & 0.54 \\
\hline Yancheng & 305 & 190 & 0.44 & 0.31 & 1.61 & 0.54 \\
\hline Yangzhou & 206 & 118 & 0.80 & 0.39 & 1.75 & 0.59 \\
\hline Zhenjiang & 148 & 70 & 0.80 & 0.59 & 2.11 & 0.73 \\
\hline Taizhou & 169 & 93 & 0.81 & 0.43 & 1.82 & 0.62 \\
\hline Suqian & 196 & 118 & 0.65 & 0.34 & 1.66 & 0.56 \\
\hline
\end{tabular}

TABLE 3: Classification of coordination levels.

\begin{tabular}{lcll}
\hline Level & Coordination & Definition & \begin{tabular}{l} 
Possible situation description \\
\hline 1
\end{tabular}$\quad 0 \sim 0.249$ \\
\hline 2 & Very low & $\begin{array}{l}\text { Least preferred tourism destination in the province, lacks attractions and thus } \\
\text { potential customers. }\end{array}$ \\
\hline 3 & Low & $\begin{array}{l}\text { Basically, not a tourism destination, with only a few notable attractions and } \\
\text { services; the local population is reluctant to travel. }\end{array}$ \\
\hline 4 & $0.400 \sim 0.599$ & Medium & $\begin{array}{l}\text { Average economy, basically not reliant on tourism but contains some level of a } \\
\text { hard-core tourism industry that supplements the overall economy. }\end{array}$ \\
\hline 5 & High & $\begin{array}{l}\text { Tourism industry is promising; residents are highly motivated to travel, but the area } \\
\text { still needs time and further investment to grow. }\end{array}$ \\
\hline
\end{tabular}


TABLE 4: Degrees of coordination and supply-demand balances for municipalities of Jiangsu province.

\begin{tabular}{|c|c|c|c|c|c|}
\hline Municipality & Supply & Demand & Coupling & Coordination & Classification \\
\hline Nanjing & 0.897 & 0.709 & 0.993 & 0.893 & $\begin{array}{l}\text { Very high coordination } \\
\text { Oversupplied }\end{array}$ \\
\hline Wuxi & 0.434 & 0.670 & 0.977 & 0.734 & $\begin{array}{l}\text { High coordination } \\
\text { Undersupplied }\end{array}$ \\
\hline Xuzhou & 0.220 & 0.237 & 0.999 & 0.478 & $\begin{array}{l}\text { Medium coordination } \\
\text { Balanced supply-demand }\end{array}$ \\
\hline Changzhou & 0.271 & 0.346 & 0.993 & 0.554 & $\begin{array}{l}\text { Medium coordination } \\
\text { Undersupplied }\end{array}$ \\
\hline Suzhou & 0.797 & 0.938 & 0.997 & 0.930 & $\begin{array}{l}\text { Very high coordination } \\
\text { Undersupplied }\end{array}$ \\
\hline Nantong & 0.315 & 0.311 & 1.000 & 0.560 & $\begin{array}{l}\text { Medium coordination } \\
\text { Balanced supply-demand }\end{array}$ \\
\hline Lianyungang & 0.199 & 0.093 & 0.932 & 0.369 & $\begin{array}{l}\text { Low coordination } \\
\text { Oversupplied }\end{array}$ \\
\hline Huai'an & 0.124 & 0.085 & 0.982 & 0.320 & $\begin{array}{l}\text { Low coordination } \\
\text { Oversupplied }\end{array}$ \\
\hline Yancheng & 0.152 & 0.181 & 0.996 & 0.408 & $\begin{array}{l}\text { Medium coordination } \\
\text { Balanced supply-demand }\end{array}$ \\
\hline Yangzhou & 0.286 & 0.285 & 1.000 & 0.534 & $\begin{array}{l}\text { Medium coordination } \\
\text { Balanced supply-demand }\end{array}$ \\
\hline Zhenjiang & 0.038 & 0.155 & 0.795 & 0.277 & $\begin{array}{l}\text { Low coordination } \\
\text { Undersupplied }\end{array}$ \\
\hline Taizhou & 0.084 & 0.142 & 0.968 & 0.331 & $\begin{array}{l}\text { Low coordination } \\
\text { Undersupplied }\end{array}$ \\
\hline Suqian & 0.002 & 0.004 & 0.938 & 0.051 & $\begin{array}{l}\text { Very low coordination } \\
\text { Undersupplied }\end{array}$ \\
\hline
\end{tabular}

TABLE 5: Regression results.

\begin{tabular}{lcccccc}
\hline Data set & $R^{2}$ & $F$ & $\mathrm{df}_{1}$ & $\mathrm{df}_{2}$ & Sig. & Equation \\
\hline CD-coordination & 0.330 & 2.463 & 2 & 10 & 0.135 & $y=3.092 x 2-3.597 x+1.376$ \\
$\alpha$-Coordination & 0.111 & 0.652 & 2 & 10 & 0.555 & $y=-9.443 x 2+8.838 x-1.474$ \\
$\beta$-Coordination & 0.104 & 0.580 & 2 & 10 & 0.578 & $y=-2.564 x 2+9.751 x-8.682$ \\
$\gamma$-Coordination & 0.146 & 0.854 & 2 & 10 & 0.454 & $y=-24.306 x 2+31.360 x-9.510$ \\
\hline
\end{tabular}

opposite may not be as obvious. The regression results are presented in Table 5.

The results clearly show that the network indices are not highly related to the degree of coordination; even the most highly related parameter, the corridor density, has a relatively low $R^{2}$ value. This demonstrates that the supplydemand balance of tourism is not significantly affected by the spatial structure of the traffic network. Traffic network within a region itself, after all, mainly serves the travel of economical and domestic affair purposes; tourism travels are but a small portion in these major purposes, not to mention that the existence of road connection does not necessarily have equal travel potential. Traffic flow data for the traffic entering and exiting on the nodes of network are thus required to determine the impact of traffic structure on the supply-demand balance of tourism and to provide advice concerning the potential future of the tourism industry in a given location.

\section{Conclusions}

This research established a traffic connection network, calculated its topological attributes, and analyzed the supplydemand balance of tourism in the region to determine if the spatial distribution of regional traffic network would impact regional tourism supply-demand balance and if further data concerning traffic flow are required for the analysis and direction of the development and construction of local traffic infrastructure to support the tourism industry.

The characterization of traffic network attributes indicates that, although there is a considerable level of connections among roads, the number of circuits of roads is limited and travel between nodes likely requires the traveler to follow the same path for their return or possibly even take a detour. The tourism coordination results reveal that 3 cities are at higher branches of coordination condition, while 5 are 
relatively low compared to provincial average. A majority of cities in Jiangsu are undersupplied, indicating the necessity of expanding tourism investment to satiate local demand.

The regression analysis indicates that the topological attributes of the traffic network are not directly related to the coordination of the supply-demand balance of tourism, which is possibly because traffic network is mainly built to serve purposes other than tourism, and presence of traffic connections may have enormous differences in travel potential. Therefore, detailed information concerning the traffic flow at network nodes is required to further analyze the relationship between traffic spatial structure and the development of a supply-demand balance for tourism, as well as to provide advice based on actual traffic situations and tendencies rather than merely tourism benefits.

Because traffic flow data will be needed for future research, the next step will be to collect data from city entrance surveillance systems. However, these data will only be available for several major cities, whereas small towns and villages are not establishing any such systems in the near future. An initial solution would be to establish a highly simplified network that only includes major cities, and a further solution could be the use of portable wireless sensors to establish a temporary network with a properly designed monitoring schedule or simply (hire locals) count traffic in a sample time period if it is a small village, thus gradually establishing a provincial network.

The results from this case study elucidated a limitation of this research and confirmed the need to include traffic flow data in future research efforts. In addition, data for only a single sample year were used to establish the connection network as well as the degree of coordination. The data were collected on prefectural basis; thus, only 13 sets of data were calculated, which is a relatively small sample size for regression analysis. Further research should collect data on a county basis and incorporate more years.

\section{Conflict of Interests}

The author declares that there is no conflict of interests regarding the publication of this paper.

\section{References}

[1] H. L. Liu, T. Feng, and X. Yang, "The energy requirements and carbon dioxide emissions of tourism industry of Western China: a case of Chengdu city," Renewable and Sustainable Energy Reviews, vol. 15, no. 6, pp. 2887-2894, 2011.

[2] Q. S. Ge, J. H. Dai, H. L. Liu et al., "The effect of climate change on the fall foliage vacation in China," Tourism Management, vol. 38, pp. 80-84, 2012.

[3] H. L. Liu, X. Yang, Z. Qiao, and H. L. Liu, "Algorithms and applications in grass growth monitoring," Abstract and Applied Analysis, vol. 2013, Article ID 508315, 7 pages, 2013.

[4] W. Gronau and A. Kagermeier, "Key factors for successful leisure and tourism public transport provision," Journal of Transport Geography, vol. 15, no. 2, pp. 127-135, 2007.

[5] C. R. Goeldner and J. R. Brent Ritchie, Tourism: Principles, Practices, Philosophies, Wiley, New York, NY, USA, 2009.
[6] S. Formica and M. Uysal, "Destination attractiveness based on supply and demand evaluations: an analytical framework," Journal of Travel Research, vol. 44, no. 4, pp. 418-430, 2006.

[7] H. Haken, Synergetics, An Introduction: Nonequilibrium Phase Transitions and Self-Organization in Physics, Chemistry, And Biology, Springer, New York, NY, USA, 1983.

[8] H. Hermann, Advanced Synergetics: Instability Hierarchies of Self-Organizing Systems and Devices Springer Series in Synergetics, Springer, New York, NY, USA, 1983.

[9] H. Haken, Synergetics: Introduction and Advanced Topics, Springer, New York, NY, USA, 2004.

[10] Y. M. Wu, D. F. Liang, Z. H. Zhang et al., "Coordinative degree model of environment-economy system and its application," China Population, Resources and Environment, vol. 6, no. 2, pp. 47-50, 1996 (Chinese).

[11] C. Gunn and T. Var, Tourism Planning: Basics Concepts Cases, Routledge, New York, NY, USA, 4th edition, 2002.

[12] B. H. Wu, "Tourism system: a explanation on tourism activities and tourism science," Tourism Tribune, vol. 14, no. 1, pp. 21-25, 1998 (Chinese).

[13] T. Bieger and A. Wittmer, "Air transport and tourism-perspectives and challenges for destinations, airlines and governments," Journal of Air Transport Management, vol. 12, no. 1, pp. 40-46, 2006.

[14] R. Culpan, "International tourism model for developing economies," Annals of Tourism Research, vol. 14, no. 4, pp. 541-552, 1987.

[15] B. Prideaux, "The role of the transport system in destination development," Tourism Management, vol. 21, no. 1, pp. 53-63, 2000.

[16] P. Hagget, A. D. Cliff, and A. Fry, Locational Analysis in Human Geography, Wiley, New York, NY, USA, 2nd edition, 1997.

[17] P. Hagget and R. J. Chorley, Network Analysis in Geography, vol. 348, Edward Arnold, London, UK, 1972.

[18] S. Q. Wei and T. F. Jia, "A spatial structure analysis and optimization of tourism resources in Hangzhou," Tourism Science, vol. 19, no. 6, pp. 21-26, 2005.

[19] R. H. Qi and S. D. Xiong, "Assessment of the current status \& planning of the green space system of Chongming Island using landscape metrics and network analysis methods," Ecology Science, vol. 26, no. 3, pp. 208-214, 2007 (Chinese).

[20] G. Bagler, "Analysis of the airport network of India as a complex weighted network," Physica A: Statistical Mechanics and its Applications, vol. 387, no. 12, pp. 2972-2980, 2008.

[21] D. G. Pearce, "Spatial patterns of package tourism in Europe," Annals of Tourism Research, vol. 14, no. 2, pp. 183-201, 1987.

[22] J. Weng and K. Z. Yang, "Spatial structure of tourism system: a spatial model for monopolistic competition with asymmetry," System Engineering Theory and Practice, vol. 27, no. 2, pp. 76-82, 2007.

[23] F. Lu, C. H. Zhou, and Q. Wan, "Implementation of a featurebased non-planar database for urban traffic networks," Acta Geodaetica et Cartographica Sinica, vol. 31, no. 2, pp. 182-186, 2002 (Chinese).

[24] M. Beuthe, B. Jourquin, J. Geerts, and C. Koul À Ndjang'Ha, "Freight transportation demand elasticities: a geographic multimodal transportation network analysis," Transportation Research Part E: Logistics and Transportation Review, vol. 37, no. 4, pp. 253-266, 2001.

[25] B. Entwisle, R. R. Rindfuss, S. J. Walsh, T. P. Evans, and S. R. Curran, "Geographic information systems, spatial network 
analysis, and contraceptive choice," Demography, vol. 34, no. 2, pp. 171-187, 1997.

[26] P. Y. Zhang, F. Su, H. Li, and R. H. Qiu, "Coordination degree of urban population, economy, space, and environment in Shenyang since 1990," China Population Resources and Environment, vol. 18, no. 2, pp. 115-119, 2008.

[27] Y. F. Li, Y. Li, Y. Zhou et al., "Investigation of a coupling model of coordination between urbanization and the environment," Journal of Environmental Management, vol. 98, pp. 127-133, 2012.

[28] Z. B. Wang, C. L. Fang, S. W. Cheng et al., "Evolution of coordination degree of eco-economic system and early-warning in the Yangtze River Delta," Journal of Geographical Sciences, vol. 23, no. 1, pp. 147-162, 2013. 


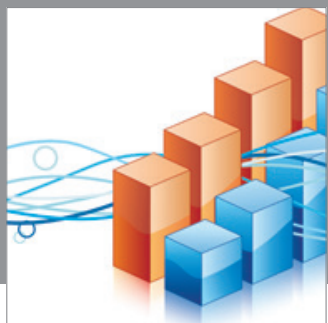

Advances in

Operations Research

mansans

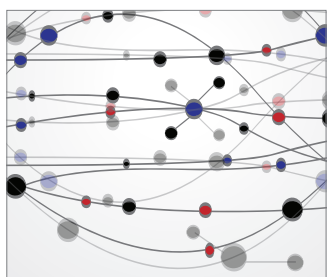

The Scientific World Journal
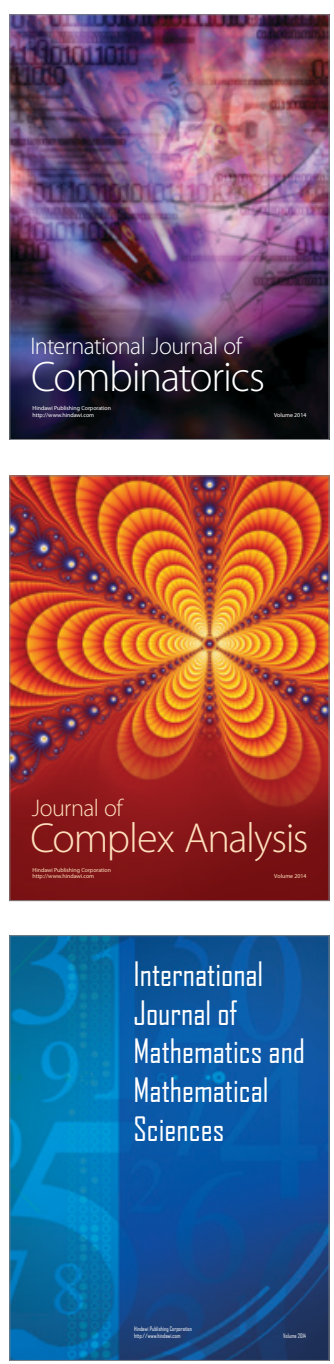
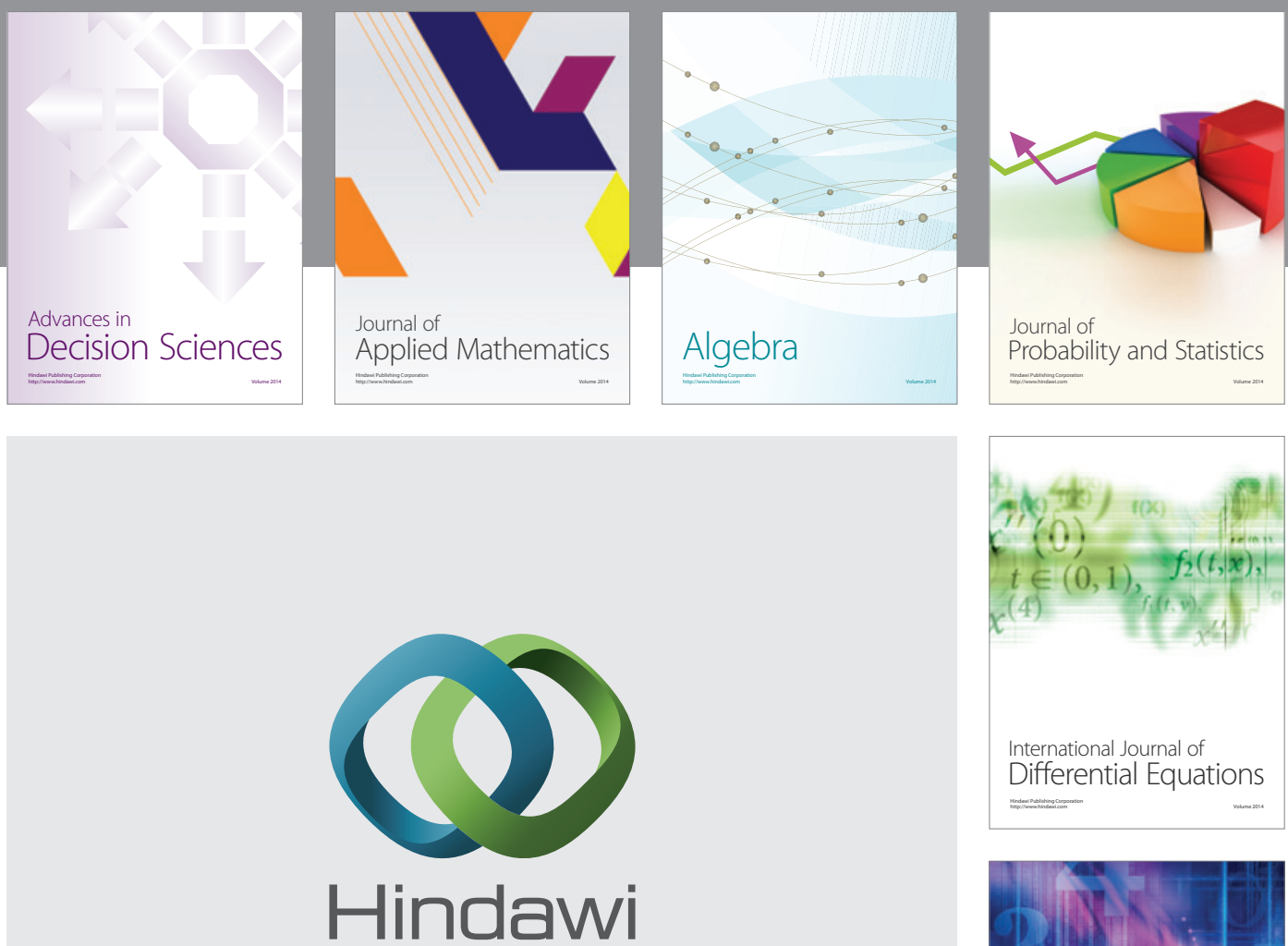

Submit your manuscripts at http://www.hindawi.com
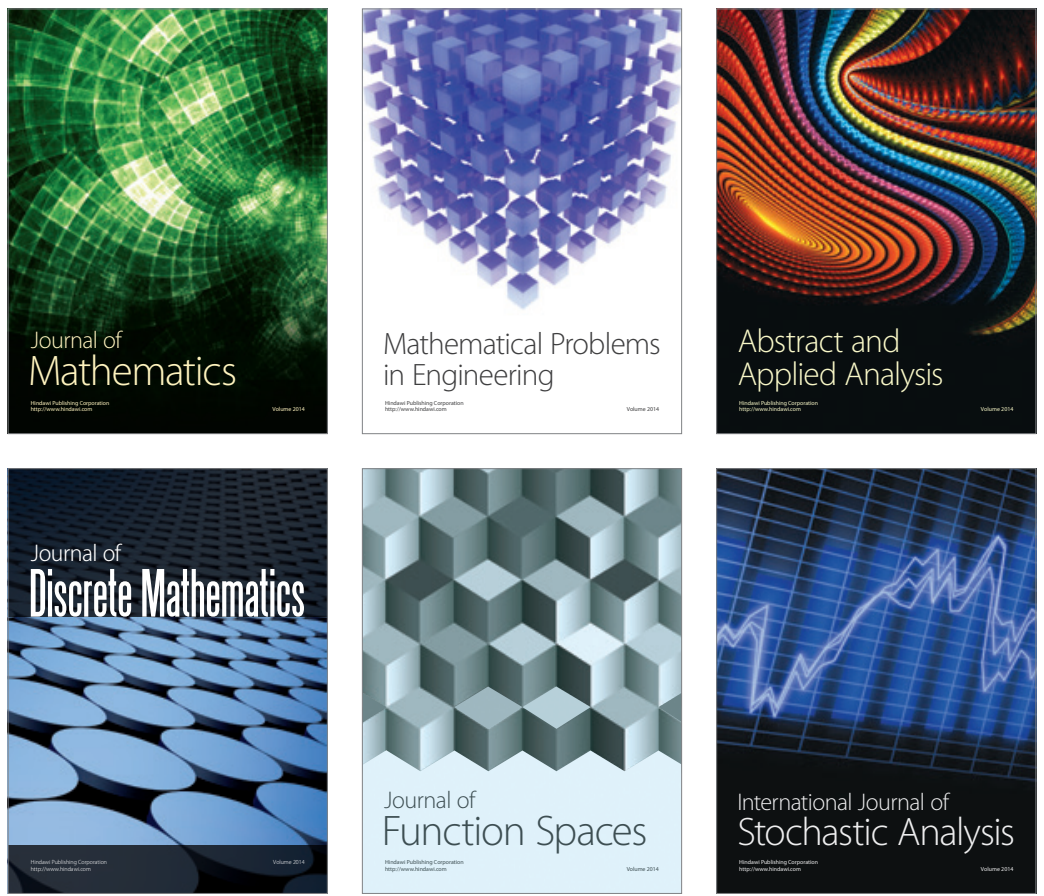

Journal of

Function Spaces

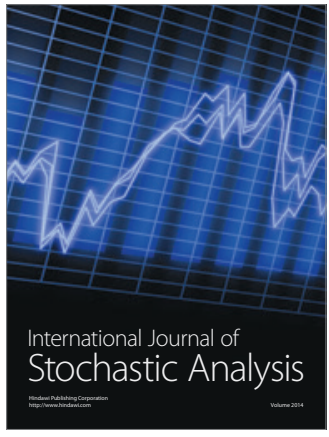

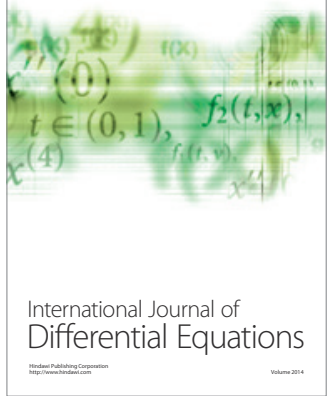
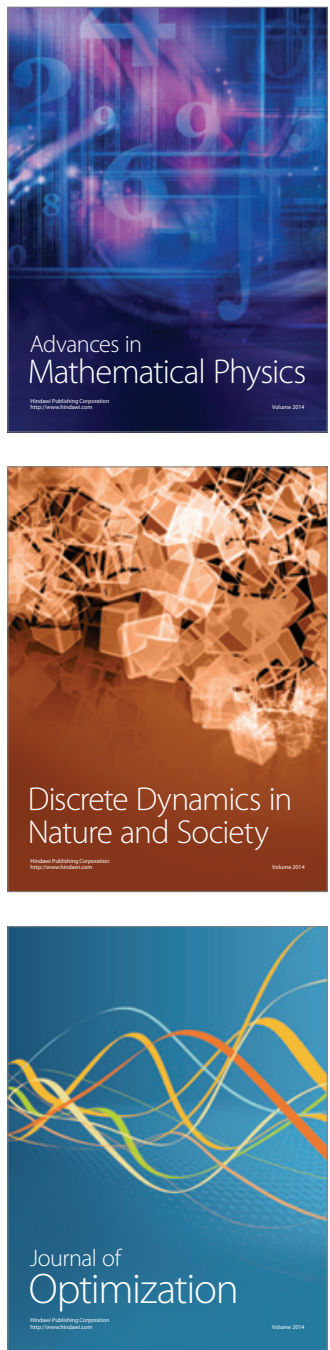